\title{
Periodontal treatment in pregnant women improves periodontal disease but does not alter rates of preterm birth
}

\author{
Does periodontal treatment during pregnancy reduce the rate of preterm births?
}

\author{
Michalowicz BS, Hodges JS, DiAngelis AJ, et al. Treatment of peri- \\ odontal disease and the risk of preterm birth. N Engl J Med 2006; \\ 355:1885-1894
}

Design This was a multicentre randomised controlled trial (RCT).
Intervention Pregnant women were divided into two groups. Those
to whom dental treatment was given had up to four visits of periodon-
tal scaling and root planing using ultrasonic and hand instruments,
with local anaesthesia as needed. Treatment recipients were also given
instruction in oral hygiene and monthly tooth polishing and re-instruc-
tion in oral hygiene, with scaling and planing as needed. Control patients
received only a brief oral examination at monthly follow-ups but attend-
ed the same number of visits as the treatment group. Patients in the con-
trol group were offered the same periodontal therapy after delivery.
Outcome measure The primary outcome was gestational age at
delivery. Secondary outcomes included birth weight, the proportion of
infants who were small for gestational age, Apgar scores, and admissions
to a neonatal intensive care unit. Results Preterm birth (before 37 weeks of gestation) occurred in 49 out of 407 women (12.0\%) in the treatment group (resulting in 44 live births) and in 52 out of 405 women (12.8\%) in the control group (resulting in 38 live births). Although periodontal treatment improved periodontitis measures $(P<0.001)$, it did not significantly alter the risk of preterm delivery [P 0.70; hazard ratio for treatment group versus control group, 0.93 ; 95\% confidence interval $(\mathrm{Cl}), 0.63-1.37]$. There were no significant differences between the treatment and control groups in mean babies' birth weights (3239 g versus $3258 \mathrm{~g}$; P 0.64) or in the rate of delivery of infants who were small for gestational age ( $12.7 \%$ versus $12.3 \%$; odds ratio, 1.04 ; $95 \% \mathrm{Cl}, 0.68-1.58)$. There were five spontaneous abortions or stillbirths in the treatment group, compared with 14 in the control group (P 0.08).

Conclusions Treatment of periodontitis in pregnant women improves periodontal disease and is safe but does not significantly alter rates of preterm birth, low birth weight or foetal growth restriction.

\section{Commentary}

Periodontitis treatment during pregnancy does not significantly change the rates of preterm birth, low birth weight, foetal growth restriction, or pre-eclampsia. Preterm delivery, low birth weight and pre-eclampsia represent severe problems in neonatology and the potential benefits of interventions aiming to reduce their occurrence are substantial. The association between periodontitis and poor pregnancy outcomes has been documented in the literature but several risk factors for poor pregnancy outcomes, such as socioeconomic factors, smoking, diabetes and stress, have also been consistently associated with periodontitis. Observational research on the topic has not been able to provide reliable answers to the ques-

Address for correspondence: BS Michalowicz, Department of Developmental and Surgical Sciences, University of Minnesota, Minneapolis, USA. E-mail:

micha002@umn.edu tion: does periodontitis cause these poor pregnancy outcomes?

The main problem has been the poor (or lack of) consideration of confounders in these studies. Although restricting the study groups to women who were never smokers solves the issue of smoking, ${ }^{1}$ there is no way to effectively cope with the pervasive effects of socioeconomic inequalities in observational research because they follow gradients, ${ }^{2}$ making any attempt to control for confounding problematic.

The most suitable study design to address this problem is the RCT. The results of two previous intervention studies suggest that the use of periodontal treatment may be beneficial, ${ }^{3-4}$ but issues such as the lack of balance between groups for known confounders at baseline, the selection of study participants and poor reporting make this evidence unconvincing ${ }^{5-6}$

This study is a well-designed and adequately reported multicentre RCT that addresses clear and focused questions. The main relevant, clinical outcomes were considered; there was good compliance through the study; and all patients who entered the trial were properly accounted for at study conclusion. The authors assessed whether nonsurgical periodontal treatment in pregnant women reduced the risk of delivery before 37 weeks and whether it resulted in a greater birth weight and a reduced proportion of infants who were small for gestational age. The groups were similar at the start of the trial and patients not complying with the research schedule were still considered in the treatment groups to which they were allocated in order to perform intention-to-treat analysis. Adjusted analyses considered the study design and baseline risk factors.

Periodontal condition improved after the intervention but it did not significantly affect the risk of preterm delivery, low birth weight, foetal growth restriction or pre-eclampsia. The conclusions of the authors are consistent with the analyses performed and the study provides the most reliable evidence available on this topic to date to be determined.

\section{Practice point}

Periodontal therapy does not reduce the occurrence of poor pregnancy outcomes.

\section{Rodrigo López}

Department of Community Oral Health and Paediatric Dentistry, Faculty of Health Sciences, University of Aarhus, Aarhus, Denmark

1. Farrell S, Ide M, Wilson RF. The relationship between maternal periodontitis, adverse pregnancy outcome and miscarriage in never smokers. I Clin Periodontol 2006; 33:115-120.

2. Lopez R, Fernández O, Baelum V. Social gradients in periodontal disease among adolescents. Community Dent Oral Epidemiol 2006; 34:184-196.

3. Jeffcoat MK, Hauth JC, Geurs NC, et al. Periodontal disease and preterm birth: results of a pilot intervention study. | Periodontol 2003; 74:1214-1218.

4. Lopez NI, Smith PC, Gutiérrez I. Periodontal therapy may reduce the risk of preterm low birth weight in women with periodontal disease: a randomized controlled trial. Periodontol 2002; 73:911-924.

5. Matthews D. Treatment of periodontal disease may significantly reduce susceptible women's risk of delivering preterm low-birthweight babies. J Evid Base Dent Pract 2003; 3:86-87.

6. Davenport E. Treatment of periodontitis reduces the risk of spontaneous preterm birth. J Evid Base Dent Pract 2004; 4:220-221.

Evidence-Based Dentistry (2007) 8, 5-6. doi:10.1038/sj.ebd.6400486 